Homology, Homotopy and Applications, vol.4(1), 2002, pp.1-23

\title{
COHOMOLOGY OF GROUPS WITH OPERATORS
}

\section{A. M. CEGARRA, J. M. GARCÍA-CALCINES And J. A. ORTEGA}

\author{
(communicated by Hvedri Inassaridze)
}

\begin{abstract}
Well-known techniques from homological algebra and algebraic topology allow one to construct a cohomology theory for groups on which the action of a fixed group is given. After a brief discussion on the modules to be considered as coefficients, the first section of this paper is devoted to providing some definitions for this cohomology theory and then to proving that they are all equivalent. The second section is mainly dedicated to summarizing certain properties of this equivariant group cohomology and to showing several relationships with the ordinary group cohomology theory.
\end{abstract}

\section{Introduction}

If $\Gamma$ is a group, then a $\Gamma$-group is a group $G$ endowed with a $\Gamma$-action by automorphisms. Because $\Gamma$-groups arise in nature of numerous algebraic, geometric and topological problems, it should be clear that their study, as algebraic objects in their own right, is a subject of interest. However, so far the authors know, there is no good source of information about $\Gamma$-groups in the literature, and particularly there is no systematic study on a specific cohomology theory for these algebraic structures, which is the purpose of this paper. Indeed, we provide here a cohomology theory, denoted $H_{\Gamma}^{n}(G, A)$, which we think enjoys many desirable properties, to whose study the article is mainly dedicated.

We should remark that this work was originally motivated by the graded categorical groups classification problem, which was suggested by Fröhlich and Wall in $[\mathbf{1 1}]$ and that we solve in $[\mathbf{6}]$, thanks to the cohomology groups $H_{\Gamma}^{3}(G, A)$. Furthermore, the equivariant group cohomology theory, in the form introduced here, is appropriate for a systematic treatment of the general equivariant group extensions problem $[\mathbf{2 6}, \mathbf{6}]$.

The paper is organized in two sections. The first is devoted to discussing fundamental aspects concerning the definition of the cohomology groups $H_{\Gamma}^{n}(G, A)$, at the heart of which are the abelian groups of equivariant derivations $\operatorname{Der}_{\Gamma}(G, A)$. The

This work has been supported by the DGES research projects PB97-0829 and BFM2001-2886 from the Ministry of Education and Science of Spain

Received February 13, 2002; published on March 4, 2002.

2000 Mathematics Subject Classification: 18G10, 20J06, 55N25

Key words and phrases: groups with operators, cohomology.

(C) 2002, A. M. Cegarra, J. M. García-Calcines and J. A. Ortega. Permission to copy for private use granted. 
discussion includes the topological meaning of these cohomology groups and also an explicit description of Whitehead's cochain complex $C_{\Gamma}^{\bullet}(G, A)[\mathbf{2 6}]$ that makes their computation by cocycles possible. In the second section we summarize several properties of the equivariant cohomology groups that we have found and which we consider of sufficient interest to be pointed out in the article, such as equivariant versions of Hochschild-Serre results [16] for the cohomology of group extensions or those showing relevant interactions with the ordinary Eilenberg-MacLane cohomology groups.

\section{Cohomology of $\Gamma$-groups}

Throughout $\Gamma$ is a (any) fixed group, and ${ }_{\Gamma} \mathcal{G}$ denotes the category of $\Gamma$-groups, that is, the category whose objects are groups $G, H, \cdots$, enriched with a left $\Gamma$-action by automorphisms and whose morphisms are those homomorphisms $f: G \rightarrow H$ that are $\Gamma$-equivariant, in the sense that $f\left({ }^{\sigma} x\right)={ }^{\sigma} f(x), \sigma \in \Gamma, x \in G$. Such a morphism is usually termed a $\Gamma$-homomorphism. The category of abelian $\Gamma$-groups, that is, of $\Gamma$-modules, is denoted by ${ }_{\Gamma} \mathcal{A} b$.

If $G$ is a $\Gamma$-group, then ${ }_{\Gamma} \mathcal{G} / G$ is the category whose objects are the $\Gamma$-homomorphisms with range $G$ and whose morphisms are the usual commutative triangles. We shall write objects and morphisms in ${ }_{\Gamma} \mathcal{G} / G$ as objects and morphisms in ${ }_{\Gamma} \mathcal{G}$, the morphisms to $G$ being understood.

We are going to define the cohomology of $\Gamma$-groups; hence we must first determine what the coefficients are for such a cohomology theory. To do so we recall (see $[\mathbf{3}]$ or $[\mathbf{2 3}])$ that a general notion of coefficients for the cohomology of algebraic structures says that abelian group objects in the comma category ${ }_{\Gamma} \mathcal{G} / G$ are the right coefficients for the cohomology of a $\Gamma$-group $G$. When $\Gamma=\mathbf{1}$, the trivial group, it is well known that any abelian group object in $\mathcal{G} / G$ is isomorphic to one

of the form $A \rtimes G \stackrel{p r}{\rightarrow} G$, where $A$ is a $G$-module and $A \rtimes G$ denotes the semidirect group product and, thus, the category of abelian group objects in $\mathcal{G} / G$ is equivalent to the category ${ }_{G} \mathcal{A} b$ of $G$-modules. For $\Gamma$ arbitrary, the category of abelian group objects in ${ }_{\Gamma} \mathcal{G} / G$ can be described in terms of what we call $\Gamma$-equivariant $G$-modules, which are defined next.

Definition 2.1. Let $G$ be a $\Gamma$-group. A $\Gamma$-equivariant $G$-module $A$ is a $\Gamma$-module, also denoted by $A$, enriched with a $G$-module structure by a $\Gamma$-equivariant action map $G \times A \rightarrow A$, which means that both actions of $\Gamma$ and $G$ on $A$ are compatible in the following precise sense:

$$
\left.{ }^{\sigma}\left({ }^{x} a\right)={ }^{(\sigma} x\right)\left({ }^{\sigma} a\right), \quad \sigma \in \Gamma, x \in G, a \in A .
$$

These $\Gamma$-equivariant $G$-modules are the objects of a category, denoted by

$$
{ }_{\Gamma, G} \mathcal{A} b,
$$

whose hom-sets, denoted by $\operatorname{Hom}_{\Gamma, G}(A, B)$, consist of those homomorphisms $f$ : $A \rightarrow B$ which are of both $\Gamma$ and $G$-modules, that is, such that $f\left({ }^{\sigma} a\right)={ }^{\sigma} f(a)$, $f\left({ }^{x} a\right)={ }^{x} f(a)$ for all $\sigma \in \Gamma, x \in G, a \in A$. 
Theorem 2.2. Let $G$ be a $\Gamma$-group. The following four categories are equivalent:

1. The category of abelian group objects in ${ }_{\Gamma} \mathcal{G} / G$.

2. The category ${ }_{\Gamma, G} \mathcal{A} b$, of $\Gamma$-equivariant $G$-modules.

3. The category ${ }_{G \rtimes \Gamma} \mathcal{A} b$, of $(G \rtimes \Gamma)$-modules.

4. The category of pairs $(A, \varphi: G \rightarrow A u t(A))$, in which $A$ is a $\Gamma$-module and $\varphi$ is a $\Gamma$-homomorphism, where the group Aut(A) of automorphisms of the abelian group $A$ is a $\Gamma$-group with the diagonal action, that is, with $\Gamma$-action

$$
\left({ }^{\sigma} f\right): a \mapsto^{\sigma} f\left(\sigma^{-1} a\right), \quad \sigma \in \Gamma, \quad f \in \operatorname{Aut}(A), a \in A .
$$

Proof. It is quite straightforward. Let us only note that for any $\Gamma$-equivariant $G$ module $A$, the abelian group object in ${ }_{\Gamma} \mathcal{G} / G$ it defines is given by the projection $A \rtimes G \stackrel{p r}{\rightarrow} G$, where $\Gamma$ acts on the semidirect product by ${ }^{\sigma}(a, x)=\left({ }^{\sigma} a,{ }^{\sigma} x\right)$. Furthermore, the associated $(G \rtimes \Gamma)$-action on $A$ is given by ${ }^{(x, \sigma)} a={ }^{x}\left({ }^{\sigma} a\right)$ and the corresponding $\Gamma$-homomorphism $\varphi: G \rightarrow A u t(A)$ is just the representation homomorphism, $\varphi(x)(a)={ }^{x} a$.

Since the category of $\Gamma$-equivariant $G$-modules can be identified as the category of modules over the semidirect product group, it follows that it is equational. Later on we will use the following consequences on injective $\Gamma$-equivariant $G$-modules.

Corollary 2.3. Let $G$ be a $\Gamma$-group. Then,

i) The category of $\Gamma$-equivariant $G$-modules is an abelian category that has enough injectives.

ii) If $I$ is an injective $\Gamma$-equivariant $G$-module, then $I$ is both an injective $\Gamma$-module and an injective $G$-module.

iii) If $I$ is an injective $\Gamma$-equivariant $G$-module, then $I^{G}=\left\{a \in I \mid{ }^{x} a=a, x \in G\right\}$ is an injective $\Gamma$-submodule of $I$.

Proof. ii) For any group $H$, every injective $H$-module is an injective $U$-module for any subgroup $U \subseteq H$ [15, VI, Corollary 1.4]. Since both $G$ and $\Gamma$ are subgroups of $G \rtimes \Gamma$, the assertion follows from Theorem 2.2.

iii) For any $\Gamma$-equivariant $G$-module $A, A^{G}=\left\{a \in A \mid{ }^{x} a=a, x \in G\right\}$ is a $\Gamma$ submodule of $A$ since, for every $a \in A^{G}, \sigma \in \Gamma$ and $x \in G,{ }^{x}\left({ }^{\sigma} a\right) \stackrel{(1)}{=} \sigma\left({\left({ }^{-1} x\right)} a\right)={ }^{\sigma} a$, whence ${ }^{\sigma} a \in A^{G}$. Therefore, we have the functor $(-)^{G}:{ }_{\Gamma, G} \mathcal{A} b \rightarrow{ }_{\Gamma} \mathcal{A} b$, which is right adjoint to the functor carrying each $\Gamma$-module $B$ to the $\Gamma$-equivariant $G$ module defined by itself with the trivial $G$-action ${ }^{x} b=b, x \in G$. Since this last functor preserves monomorphisms, $(-)^{G}$ preserves injectives [15, Chap. II, Proposition 10.2].

If $p: H \rightarrow G$ is a $\Gamma$-homomorphism, then on any $\Gamma$-equivariant $G$-module, $A$, can be given the $\Gamma$-equivariant $H$-module structure "via" $p$ by defining

$$
{ }^{h} a={ }^{p(h)} a, \quad a \in A, \quad h \in H,
$$

and keeping the same $\Gamma$-action on $A$. We also denote this $\Gamma$-equivariant $H$-module by $A, p$ being understood. 
Definition 2.4. Let $A$ be a $\Gamma$-equivariant $G$-module. $A \Gamma$-derivation (or crossed $\Gamma$-homomorphism) from $G$ into $A$ is a $\Gamma$-equivariant derivation from the group $G$ into the $G$-module $A$, that is, a map $d: G \rightarrow A$ with the properties

i) $d(x y)={ }^{x} d(y)+d(x), \quad x, y \in G$,

ii) $d\left({ }^{\sigma} x\right)={ }^{\sigma} d(x), \quad \sigma \in \Gamma, x \in G$.

The set $\operatorname{Der}_{\Gamma}(G, A)$, of all $\Gamma$-derivations $d: G \rightarrow A$, can be given an obvious abelian group structure. Note that if $p: H \rightarrow G$ is any $\Gamma$-homomorphism and $q: A \rightarrow B$ is any morphism of $\Gamma$-equivariant $G$-modules, then there is an induced homomorphism:

$$
p^{*} q_{*}=q_{*} p^{*}: \operatorname{Der}_{\Gamma}(G, A) \rightarrow \operatorname{Der}_{\Gamma}(H, B), \quad d \mapsto q d p .
$$

Thus, $\operatorname{Der}_{\Gamma}(-,-)$ becomes a functor from the cartesian product category of the comma category of $\Gamma$-groups over a given $\Gamma$-group $G$ by the category of $\Gamma$-equivariant $G$-modules into the category of abelian groups. Analogously as for groups (see [15, VI, Porosition 5.3], for example) we have the following

Proposition 2.5. For any $\Gamma$-homomorphism $p: H \rightarrow G$ and any $\Gamma$-equivariant $G$-module $A$, there is a natural isomorphism

$$
\operatorname{Der}_{\Gamma}(H, A) \cong \operatorname{Hom}_{\Gamma} \mathcal{G} / G(H \stackrel{p}{\rightarrow} G, A \rtimes G \stackrel{p r}{\rightarrow} G) .
$$

The category of $\Gamma$-groups is algebraic, indeed it is a variety of universal algebras, and so one can use various well-known methods to define a cohomology theory for $\Gamma$-groups. Next we consider five definitions of the cohomology of a $\Gamma$-group $G$ with values in a $\Gamma$-equivariant $G$-module $A$; the first four definitions develop the subject from the perspective of homological algebra, while the last one shows that the subject can be considered part of algebraic topology. Our main result here is to prove that these five definitions are equivalent.

1. $H_{\Gamma}^{n}(G, A)$ as the derived functor of derivations.

For any $\Gamma$-group $G$, the functor $\operatorname{Der}_{\Gamma}(G,-)$ is a left exact functor from the category of $\Gamma$-equivariant $G$-modules to the category of abelian groups. By Corollary 2.3 , the category of $\Gamma$-equivariant $G$-modules is abelian and has enough injectives, so one can form the right derived functors of $\operatorname{Der}_{\Gamma}(G,-)$. These are, by definition, the cohomology groups of $G$ (cf. $[\mathbf{2}])$. More precisely, making a shift in dimension motivated both by comparison with the usual Eilenberg-MacLane cohomology of groups (see Theorem 3.5) and by (12), the first definition for the cohomology of a $\Gamma$-group $G$ with coefficients in a $\Gamma$-equivariant $G$-module $A$ is

$$
H_{\Gamma}^{n}(G, A)=R^{n-1} \operatorname{Der}_{\Gamma}(G,-)(A), \quad n \geqslant 1,
$$

and we take $H_{\Gamma}^{0}(G, A)=0$.

2. $H_{\Gamma}^{n}(G, A)$ by cocycles: the Whitehead complex $C_{\Gamma}^{\bullet}(G, A)$.

Both for theoretical and computational interests, it is appropriate to have an explicit description of a manageable cochain complex $C_{\Gamma}^{\bullet}(G, A)$ to compute the 
cohomology groups $H_{\Gamma}^{n}(G, A)$. This is the aim of our second definition for the cohomology of a $\Gamma$-group $G$ with values in a $\Gamma$-equivariant $G$-module $A$.

First we shall recall that for any group $G$ and any $G$-module $A$, the ordinary cohomology groups $H^{n}(G, A)$ can be computed as the cohomology groups of the abelian group positive-complex $C^{\bullet}(G, A)$, in which each $C^{p}(G, A)$ consists of all maps $f: G^{p} \rightarrow A$ such that $f\left(x_{1}, \cdots, x_{p}\right)=0$ whenever $x_{i}=1$ for some $i=1, \cdots, p$, and the coboundary $\partial: C^{p-1}(G, A) \rightarrow C^{p}(G, A)$ is defined by $(\partial f)\left(x_{1}, \cdots, x_{p}\right)=$ ${ }^{x_{1}} f\left(x_{2}, \cdots, x_{p}\right)+\sum_{i=1}^{p-1}(-1)^{i} f\left(x_{1}, \cdots, x_{i} x_{i+1}, \cdots, x_{p}\right)+(-1)^{p} f\left(x_{1}, \cdots, x_{p-1}\right)$, (see $[9])$.

Suppose now that $G$ is a $\Gamma$-group and that $A$ is a $\Gamma$-equivariant $G$-module. Then, every abelian group $C^{p}(G, A)$ has a $\Gamma$-module structure by the diagonal action

$$
\left({ }^{\sigma} f\right)\left(x_{1}, \cdots, x_{p}\right)={ }^{\sigma} f\left(\sigma^{-1} x_{1}, \cdots,{ }^{\sigma^{-1}} x_{p}\right), \quad \sigma \in \Gamma, f \in C^{p}(G, A), \quad x \in G,
$$

and the coboundaries $\partial: C^{p}(G, A) \rightarrow C^{p+1}(G, A)$ become $\Gamma$-module homomorphisms, as is easily proved thanks to equalities (1): $\left.{ }^{\sigma}\left({ }^{x} a\right)={ }^{\sigma}{ }^{\sigma} x\right)\left({ }^{\sigma} a\right)$. Thus, $C^{\bullet}(G, A)$ is a cochain complex of $\Gamma$-modules, and then one can form a double cochain complex $C_{\Gamma}^{\bullet \bullet}(G, A)$ in which $C_{\Gamma}^{p, q}(G, A)=C^{q}\left(\Gamma, C^{p+1}(G, A)\right), \quad p, q \geqslant 0$. We shall define the complex $C_{\Gamma}^{\bullet}(G, A)$ to be, up to a shift dimension and an obvious isomorphism, the total complex of the bicomplex $C_{\Gamma}^{\bullet \bullet}(G, A)$, that is, $C_{\Gamma}^{0}(G, A)=0$ and $C_{\Gamma}^{n}(G, A)=\operatorname{Tot}^{n-1}\left(C_{\Gamma}^{\bullet \bullet}(G, A)\right)$ for $n \geqslant 1$.

More precisely, the elements of $C_{\Gamma}^{n}(G, A)$, related as $n$-cochains of the $\Gamma$-group $G$ with coefficients in $A$, are the maps

$$
f: \bigcup_{p+q=n-1} G^{p+1} \times \Gamma^{q} \longrightarrow A,
$$

which are normalized in the sense that $f\left(x_{1}, \cdots, x_{p+1}, \sigma_{1}, \cdots, \sigma_{q}\right)=0$ whenever $x_{i}=1$ or $\sigma_{j}=1$ for some $i=1, \cdots, p+1$ or $j=1, \cdots, q$. The cochain complex $C_{\Gamma}^{\bullet}(G, A)=\left\{C_{\Gamma}^{n}(G, A), \partial\right\}$ is then defined by the coboundary

$$
\partial: C_{\Gamma}^{n}(G, A) \rightarrow C_{\Gamma}^{n+1}(G, A) \quad n \geqslant 1,
$$

given by the formula

$$
\begin{aligned}
&(\partial f)(\underline{x}, \underline{\sigma})={ }^{\sigma_{1}} f\left(\underline{x}, \sigma_{2}, \cdots, \sigma_{q}\right)+\sum_{i=1}^{q-1}(-1)^{i} f\left(\underline{x}, \sigma_{1}, \cdots, \sigma_{i} \sigma_{i+1}, \cdots, \sigma_{q}\right)+ \\
&(-1)^{q} f\left({ }^{\sigma_{q}} x_{1}, \cdots,{ }^{\sigma_{q}} x_{p+1}, \sigma_{1}, \cdots, \sigma_{q-1}\right)+ \\
&(-1)^{q}\left[{ }^{\left({ }^{\sigma_{1} \cdots \sigma_{q}} x_{1}\right)} f\left(x_{2}, \cdots, x_{p+1}, \underline{\sigma}\right)+\right. \\
&\left.\sum_{j=1}^{p}(-1)^{j} f\left(x_{1}, \cdots, x_{j} x_{j+1}, \cdots x_{p+1}, \underline{\sigma}\right)+(-1)^{p+1} f\left(x_{1}, \cdots, x_{p}, \underline{\sigma}\right)\right],
\end{aligned}
$$

where $(\underline{x}, \underline{\sigma})=\left(x_{1}, \cdots, x_{p+1}, \sigma_{1}, \cdots, \sigma_{q}\right)$ and $p+q=n$.

Then, the second definition is

$$
H_{\Gamma}^{n}(G, A)=H^{n}\left(C_{\Gamma}^{\bullet}(G, A)\right), \quad n \geqslant 0 .
$$


3. $H_{\Gamma}^{n}(G, A)$ as a cotriple cohomology.

The cotriple cohomology was developed for any tripleable (=monadic) category by Beck [3] and Barr and Beck [1], who showed that most of the cohomology theories in Algebra are particular instances of cotriple cohomology, including the EilenbergMacLane cohomology of groups. The category of $\Gamma$-groups is tripleable over $\mathcal{S}$ ets $[\mathbf{2 0}]$, that is, the underlying functor from ${ }_{\Gamma} \mathcal{G}$ to the category of sets induces a triple $\mathbb{T}$ on Sets such that an Eilenberg-Moore's $\mathbb{T}$-algebra is just a $\Gamma$-group. It is then natural to specialize cotriple cohomology for $\Gamma$-groups, which leads to our third definition for the cohomology of a $\Gamma$-group $G$ with coefficients in a $\Gamma$-equivariant $G$-module $A$.

Given a $\Gamma$-group $G$, the resulting cotriple $(\mathbb{G}, \varepsilon, \delta)$ in the comma category ${ }_{\Gamma} \mathcal{G} / G$ is as follows. For each $\Gamma$-group $H \stackrel{\varphi}{\rightarrow} G$ over $G, \mathbb{G}(H \stackrel{\varphi}{\rightarrow} G)=\mathbb{F} H \stackrel{\bar{\varphi}}{\rightarrow} G$, where $\mathbb{F} H$ is the free $\Gamma$-group on the set $H$ (= free group on the set $H \times \Gamma$ with the $\Gamma$-action such that $\left.{ }^{\sigma}(h, \tau)=(h, \sigma \tau)\right)$, and $\bar{\varphi}: \mathbb{F} H \rightarrow G$ is the $\Gamma$-homomorphism such that $\bar{\varphi}(h, \sigma)={ }^{\sigma} \varphi(h)$. The counit $\delta: \mathbb{G} \rightarrow i d$ sends $H \rightarrow G$ to the $\Gamma$-homomorphism $\mathbb{F} H \rightarrow H$ such that $\delta(h, \sigma)={ }^{\sigma} h$, and the comultiplication $\varepsilon: \mathbb{G} \rightarrow \mathbb{G}^{2}$ sends $H \rightarrow G$ to the $\Gamma$-homomorphism $\mathbb{F} H \rightarrow \mathbb{F} \mathbb{F} H$ such that $\varepsilon(h, \sigma)=((h, 1), \sigma)$, $h \in H, \sigma \in \Gamma$. This cotriple produces an augmented simplicial object in the category of endofunctors in ${ }_{\Gamma} \mathcal{G} / G, \mathbb{G}_{\bullet} \stackrel{\delta}{\rightarrow} i d$, the so-called (Godement) standard resolution, which is defined by $\mathbb{G}_{n}=\mathbb{G}^{n+1}$, with face and degeneracy operators $d_{i}=\mathbb{G}^{n-i} \delta \mathbb{G}^{i}: \mathbb{G}_{n} \rightarrow \mathbb{G}_{n-1}, 0 \leqslant i \leqslant n$, and $s_{j}=\mathbb{G}^{n-j-1} \varepsilon \mathbb{G}^{j}: \mathbb{G}_{n-1} \rightarrow \mathbb{G}_{n}$, $0 \leqslant j \leqslant n-1$. Hence, for any $\Gamma$-equivariant $G$-module $A$, one obtains an augmented cosimplicial object in the category of abelian group valuated functors from ${ }_{\Gamma} \mathcal{G} / G$, $\operatorname{Der}_{\Gamma}(-, A) \rightarrow \operatorname{Der}_{\Gamma}\left(\mathbb{G}_{\bullet}, A\right)$, and then an associated cochain complex (also denoted by $\left.\operatorname{Der}_{\Gamma}\left(\mathbb{G}_{\bullet}, A\right)\right)$, obtained by taking alternating sums of the coface operators

$$
0 \rightarrow \operatorname{Der}_{\Gamma}(\mathbb{G}, A) \stackrel{\partial^{0}}{\rightarrow} \operatorname{Der}_{\Gamma}\left(\mathbb{G}^{2}, A\right) \stackrel{\partial^{1}}{\rightarrow} \operatorname{Der}_{\Gamma}\left(\mathbb{G}^{3}, A\right) \rightarrow \cdots
$$

This complex computes the cotriple cohomology of $G$ with values in $A$ :

$$
H_{\Gamma}^{n}(G, A)=H^{n-1}\left(\operatorname{Der}_{\Gamma}\left(\mathbb{G}_{\bullet}(G), A\right)\right), \quad n \geqslant 1 .
$$

4. $H_{\Gamma}^{n}(G, A)$ as a cohomology of sheaves.

It was pointed out by Quillen in $[\mathbf{2 3}]$ and by Rinehart in [24] how the Grothendieck cohomology of sheaves over a site can be used as a general method to define a cohomology theory of any kind of universal algebras. Our fourth definition of the cohomology of a $\Gamma$-group $G$ with coefficients in a $\Gamma$-equivariant $G$-module $A$ arises by specializing this method, as follows: the class of epimorphisms in ${ }_{\Gamma} \mathcal{G} / G$ is stable under composition and pullbacks (note that every epimorphism of $\Gamma$-groups is a surjective map), then we have a Grothendieck topology on ${ }_{\Gamma} \mathcal{G} / G[\mathbf{1 7}]$ if we take for coverings the families consisting of a single $\Gamma$-epimorphism $P \rightarrow H$. With this epimorphism topology on ${ }_{\Gamma} \mathcal{G} / G$, sheaves are simply left-exact (i.e., preserving coequalizers) contravariant functors, and therefore $\operatorname{Der}_{\Gamma}(-, A)$ is a sheaf of abelian groups on ${ }_{\Gamma} \mathcal{G} / G$. Hence, Grothendieck cohomology groups of ${ }_{\Gamma} \mathcal{G} / G$ with coefficients in $\operatorname{Der}_{\Gamma}(-, A)$ are defined. These are, up to a dimension shift, the cohomology 
groups of $G$ with values in $A$, that is,

$$
H_{\Gamma}^{n}(G, A)=H^{n-1}\left({ }_{\Gamma} \mathcal{G} / G, \operatorname{Der}(-, A)\right), \quad n \geqslant 1,
$$

and thus they can be computed from flask resolutions $0 \rightarrow \operatorname{Der}_{\Gamma}(-, A) \rightarrow \mathcal{F}^{0} \rightarrow$ $\mathcal{F}^{1} \rightarrow \cdots$ of the sheaf $\operatorname{Der}_{\Gamma}(-, A)$, by

$$
H_{\Gamma}^{n}(G, A)=H^{n-1}\left(0 \rightarrow \mathcal{F}^{0}(G) \rightarrow \mathcal{F}^{1}(G) \rightarrow \cdots\right), \quad n \geqslant 1 .
$$

5. $H_{\Gamma}^{n}(G, A)$ as a singular cohomology with local coefficients.

It is well known that the cohomology of a group $G$ with coefficients in a $G$ module $A$ is the singular cohomology of a classifying space for $G, B_{G}$, with local coefficients A (see [25], for example). If $G$ is a $\Gamma$-group and $A$ is a $\Gamma$-equivariant $G$-module, then $A$ is a $(G \rtimes \Gamma)$-module, according to Theorem 2.2, and so is a system of local coefficients in the classifying space of the semidirect product group $B_{G \rtimes \Gamma}$. The classifying space of the group $\Gamma, B_{\Gamma}$, is canonically a subspace (indeed, a retract) of $B_{G \rtimes \Gamma}$ with the injection map $B_{\Gamma} \hookrightarrow B_{G \rtimes \Gamma}$, induced by the inclusion homomorphism $\Gamma \hookrightarrow G \rtimes \Gamma, \sigma \mapsto(1, \sigma)$. Therefore, the singular cohomology of the pair $\left(B_{G \rtimes \Gamma}, B_{\Gamma}\right)$ with local coefficients in $A$ is defined, whence our fifth definition for the cohomology of the $\Gamma$-group $G$ :

$$
H_{\Gamma}^{n}(G, A)=H^{n}\left(B_{G \rtimes \Gamma}, B_{\Gamma}, A\right), \quad n \geqslant 0 .
$$

Note that when $G$ acts trivially on $A$, that is, $A$ is simply a $\Gamma$-module, then the cohomology groups $H_{\Gamma}^{n}(G, A)$ are the same as the reduced cohomology groups $\tilde{H}_{\Gamma}^{n}\left(B_{G \rtimes \Gamma}, A\right)$ defined by Goerss and Jardine in [13, VI, Sect. 4].

The main result of this section is presented below.

Theorem 2.6. The definitions of $H_{\Gamma}^{n}(G, A)$ given above are equivalent.

Proof. Equivalence of (3) and (12): since both $B_{G \rtimes \Gamma}$ and $B_{\Gamma}$ have a unique 0cell, it follows that $H_{\Gamma}^{0}\left(B_{G \rtimes \Gamma}, B_{\Gamma}, A\right)=0$. The proof consists in proving that the functors $H^{n}\left(B_{G \rtimes \Gamma}, B_{\Gamma},-\right), n \geqslant 1$, form a connected sequence defining a right satellite of $\operatorname{Der}_{\Gamma}(G,-)$. Since any short exact sequence of local coefficients in $B_{G \rtimes \Gamma}$ provides a corresponding long exact sequence in the relative singular cohomology groups, $\left\{H^{n}\left(B_{G \rtimes \Gamma}, B_{\Gamma},-\right), n \geqslant 1\right\}$ is therefore a connected sequence of functors, and it then suffices to prove that $H^{1}\left(B_{G \rtimes \Gamma}, B_{\Gamma}, A\right) \cong \operatorname{Der}_{\Gamma}(G, A)$ and that $H^{n}\left(B_{G \rtimes \Gamma}, B_{\Gamma}, A\right)=0$ for $n \geqslant 2$ whenever $A$ is an injective $\Gamma$-equivariant $G$-module. For let us note that

$$
H^{n}\left(B_{G \rtimes \Gamma}, B_{\Gamma}, A\right)=H^{n}\left(C^{\bullet}(G \rtimes \Gamma, \Gamma, A)\right), n \geqslant 0,
$$

where $C^{\bullet}(G \rtimes \Gamma, \Gamma, A)=\operatorname{Ker}\left(C^{\bullet}(G \rtimes \Gamma, A) \stackrel{\text { res }}{\rightarrow} C^{\bullet}(\Gamma, A)\right)$.

Hence, $H^{1}\left(B_{G \rtimes \Gamma}, B_{\Gamma}, A\right)=Z^{1}\left(C^{\bullet}(G \rtimes \Gamma, \Gamma, A)\right)$ is the abelian group consisting of all maps $f: G \rtimes \Gamma \rightarrow A$ satisfying

i) $\quad f(1, \sigma)=0, \quad \sigma \in \Gamma$

ii) ${ }^{x}\left({ }^{\sigma} f(y, \tau)\right)-f\left(x^{\sigma} y, \sigma \tau\right)+f(x, \sigma)=0, \quad(x, \sigma),(y, \tau) \in G \rtimes \Gamma$.

We associate to each $f \in H^{1}\left(B_{G \rtimes \Gamma}, B_{\Gamma}, A\right)$ the map $d=\left.f\right|_{G}: G \rightarrow A$, which is actually a $\Gamma$-derivation from $G$ into $A$ : first observe that $d$ determines $f$ by the 
equality $f(x, \tau)=d x, x \in G, \tau \in \Gamma$. In fact, $f(x, \tau) \stackrel{i i)}{=} x f(1, \tau)+f(x, 1) \stackrel{i)}{=} f(x, 1)=$ $d x$. Thus, $i i)$ can be written in the form $d\left(x \cdot{ }^{\sigma} y\right)={ }^{x}\left({ }^{\sigma} d y\right)+d x$, which, taking $\sigma=1$, gives $d(x y)={ }^{x} d y+d x$, so $d$ is a derivation from $G$ into $A$, and taking $x=1$ gives $d\left({ }^{\sigma} y\right)={ }^{\sigma} d y$, so $d$ is $\Gamma$-equivariant. It follows that $f \mapsto d=\left.f\right|_{G}$ defines an injective map $H^{1}\left(B_{G \rtimes \Gamma}, B_{\Gamma}, A\right) \rightarrow \operatorname{Der}_{\Gamma}(G, A)$. Furthermore, for any $\Gamma$-derivation $d: G \rightarrow A$, the map $f: G \rtimes \Gamma \rightarrow A$ defined by $f(x, \sigma)=d x, x \in G, \sigma \in \Gamma$, is an element of $H^{1}\left(B_{G \rtimes \Gamma}, B_{\Gamma}, A\right)$ since

$f\left(x^{\sigma} y, \sigma \tau\right)=d\left(x^{\sigma} y\right)={ }^{x} d\left({ }^{\sigma} y\right)+d x={ }^{x}\left({ }^{\sigma} f(y, \tau)\right)+f(x, \sigma), \quad(x, \sigma),(y, \tau) \in G \rtimes \Gamma$,

whence $H^{1}\left(B_{G \rtimes \Gamma}, B_{\Gamma}, A\right) \stackrel{\cong}{\rightrightarrows} \operatorname{Der}_{\Gamma}(G, A)$ is a bijection, actually a natural isomorphism.

Suppose now that $A$ is an injective $\Gamma$-equivariant $G$-module. By Corollary 2.3, $A$ is an injective $\Gamma$-module. Since the ordinary cohomology of groups vanishes whenever the coefficients are injective modules, both complexes $C^{\bullet}(G \rtimes \Gamma, A)$ and $C^{\bullet}(\Gamma, A)$ are exact in dimensions $\geqslant 1$ and therefore the complex $\left.\operatorname{Ker}\left(C^{\bullet}(G \rtimes \Gamma, A)\right) \stackrel{\text { res }}{\rightarrow} C^{\bullet}(\Gamma, A)\right)$ is exact in dimensions $\geqslant 2$, whence $H^{n}\left(B_{G \rtimes \Gamma}, B_{\Gamma}, A\right)=0$ for all $n \geqslant 2$.

Equivalence of (7) and (12): consider the bisimplicial set $X(G)$ whose set of $p, q$ simplices is $X_{p, q}(G)=G^{p} \times \Gamma^{q}$. The vertical face and degeneracy maps are defined by those of the Eilenberg-MacLane simplicial set $K(G, 1)$, namely

$$
\begin{aligned}
& d_{i}^{v}\left(x_{1}, \cdots, x_{p}, \sigma_{1}, \cdots \sigma_{q}\right)= \begin{cases}\left(x_{2}, \cdots, x_{p}, \sigma_{1}, \cdots, \sigma_{q}\right) & \text { if } i=0 \\
\left(x_{1}, \cdots, x_{i} x_{i+1}, \cdots, x_{p}, \sigma_{1}, \cdots, \sigma_{q}\right) & \text { if } 0<i<p \\
\left(x_{1}, \cdots, x_{p-1}, \sigma_{1}, \cdots, \sigma_{q}\right) & \text { if } i=p\end{cases} \\
& s_{i}^{v}\left(x_{1}, \cdots, x_{p}, \sigma_{1}, \cdots, \sigma_{q}\right)=\left(x_{1}, \cdots, x_{i}, 1, x_{i+1}, \cdots, x_{p}, \sigma_{1}, \cdots, \sigma_{q}\right), \quad 0 \leqslant i \leqslant p,
\end{aligned}
$$

and the horizontal and degeneracy maps by those of the simplicial set $K(\Gamma, 1)$, except that $d_{q}^{h}: G^{p} \times \Gamma^{q} \rightarrow G^{p} \times \Gamma^{q-1}$ is defined by

$$
d_{q}^{h}\left(x_{1}, \cdots, x_{p}, \sigma_{1}, \cdots, \sigma_{q}\right)=\left({ }^{\sigma_{q}} x_{1}, \cdots,{ }^{\sigma_{q}} x_{p}, \sigma_{1}, \cdots, \sigma_{q-1}\right) .
$$

Observe that

$$
\operatorname{diag}(X(G)) \cong K(G \rtimes \Gamma, 1)
$$

by the simplicial map

$$
\left(x_{1}, \cdots, x_{p}, \sigma_{1}, \cdots, \sigma_{p}\right) \mapsto\left(\left({ }^{\sigma_{1} \cdots \sigma_{p}} x_{1}, \sigma_{1}\right),\left({ }^{\sigma_{2} \cdots \sigma_{p}} x_{2}, \sigma_{2}\right), \cdots,\left({ }^{\sigma_{p}} x_{p}, \sigma_{p}\right)\right) .
$$

From $X(G)$ and the given $\Gamma$-equivariant $G$-module $A$, we obtain a double cosimplicial abelian group $C^{\bullet \bullet}(X(G), A)$ in which

$$
C^{p, q}(X(G), A)=\left\{f: X_{p, q}(G) \rightarrow A\right\},
$$

the horizontal cofaces $d_{i}^{h}: C^{p, q-1}(X(G), A) \rightarrow C^{p, q}(X(G), A)$ are defined by

$$
\left(d_{i}^{h} f\right)\left(x_{1}, \cdots, x_{p}, \sigma_{1}, \cdots, \sigma_{q}\right)= \begin{cases}\sigma_{1} f\left(x_{1}, \cdots, x_{p}, \sigma_{2}, \cdots, \sigma_{q}\right) & \text { if } i=0 \\ f\left(x_{1}, \cdots, x_{p}, \sigma_{1}, \cdots, \sigma_{i} \sigma_{i+1}, \cdots, \sigma_{q}\right) & \text { if } 0<i<q \\ f\left({ }^{\sigma_{q}} x_{1}, \cdots, \sigma_{q} x_{p}, \sigma_{1}, \cdots, \sigma_{q-1}\right) & \text { if } i=q,\end{cases}
$$


and the vertical cofaces $d_{j}^{v}: C^{p-1, q}(X(G), A) \rightarrow C^{p, q}(X(G), A)$ are defined by

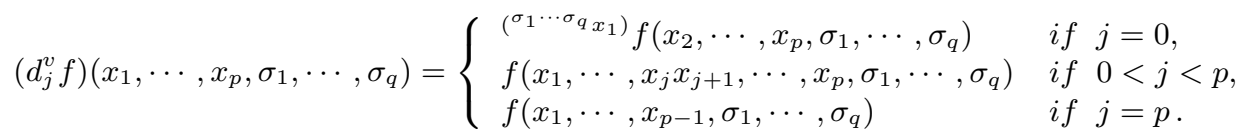

We also write $C^{\bullet \bullet}(X(G), A)$ for the associated double complex of normalized cochains, whose differentials are obtained from the face maps by taking alternating sums, and $\operatorname{Tot}\left(C^{\bullet \bullet}(X(G), A)\right)$ for the associated total complex. Observe that the isomorphism (14) induces a cochain complex isomorphism $\operatorname{diag}\left(C^{\bullet \bullet}(X(G), A)\right) \cong$ $C^{\bullet}(G \rtimes \Gamma, A)$. Then, as a result of Dold and Puppe [8], there is a quasi-isomorphism of complexes

$$
\Psi_{G}: C^{\bullet}(G \rtimes \Gamma, A) \rightarrow \operatorname{Tot}\left(C^{\bullet \bullet}(X(G), A)\right),
$$

which is natural in $G$.

Since a straightforward identification shows that the cochain complex $C_{\Gamma}^{\bullet}(G, A)$ occurs in the following commutative diagram of cochain complexes:

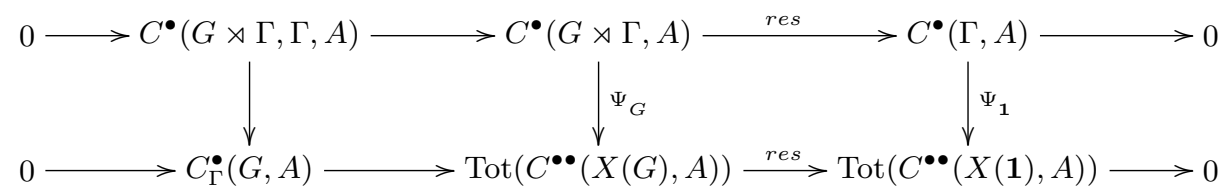

in which $\mathbf{1}$ denotes the trivial $\Gamma$-group (thus $C^{\bullet \bullet}(X(\mathbf{1}), A)$ is the double cochain complex which is the complex $C^{\bullet}(\Gamma, A)$ constant in the vertical direction), and the rows are exact, we conclude that the morphism induced by restriction of $\Psi_{G}$, $C^{\bullet}(G \rtimes \Gamma, \Gamma, A) \rightarrow C_{\Gamma}^{\bullet}(G, A)$ is a quasi-isomorphism. Then, by (13), we obtain

$$
H^{n}\left(B_{G \rtimes \Gamma}, B_{\Gamma}, A\right) \cong H^{n}\left(C_{\Gamma}^{\bullet}(G, A)\right), \quad n \geqslant 0 .
$$

Equivalence of (7) and (10): first, note that for any $\Gamma$-group over $G, H \stackrel{\varphi}{\rightarrow} G$,

$$
\operatorname{Ker}\left(C_{\Gamma}^{1}(H, A) \stackrel{\partial^{1}}{\rightarrow} C_{\Gamma}^{2}(H, A)\right)=\left\{\begin{array}{l}
f: H \rightarrow A \mid \varphi(x) f(y)-f(x y)+f(x)=0, \\
\left.\sigma f(x)-f\left({ }^{\sigma} x\right)=0\right\}=\operatorname{Der}_{\Gamma}(H, A) .
\end{array}\right.
$$

Then, the proof consists in proving that

$$
0 \rightarrow \operatorname{Der}_{\Gamma}(-, A) \rightarrow C_{\Gamma}^{1}(-, A) \rightarrow C_{\Gamma}^{2}(-, A) \rightarrow \cdots
$$

is a flask resolution of the sheaf $\operatorname{Der}_{\Gamma}(-, A)$ on ${ }_{\Gamma} \mathcal{G} / G$, that is, that (15) is exact in the category of abelian sheaves on ${ }_{\Gamma} \mathcal{G} / G$ and that every $C_{\Gamma}^{n}(-, A)$ is flask.

By [23, Chapter II, 5, Lemma 1.1] or [24, Corollary 2.5], exactness of (15) means that for any free $\Gamma$-group over $G, F$ say, $H^{n}\left(C_{\Gamma}^{\bullet}(F, A)\right)=0$ for all $n \geqslant 2$. Since we know that $H^{n}\left(C_{\Gamma}^{\bullet}(F, A)\right)=\left(R^{n-1} \operatorname{Der}_{\Gamma}(F,-)\right)(A)$, because the equivalence of definitions (3) and (7) has been proved, it suffices to prove that the functor $\operatorname{Der}_{\Gamma}(F,-)$ is exact, that is, it preserves epimorphisms. For, let $q: A \rightarrow B$ be an epimorphism of $\Gamma$-equivariant $F$-modules and let $d: F \rightarrow B$ be any $\Gamma$ derivation from $F$ into $B$. According to Proposition 2.5, the $\Gamma$-derivation $d$ defines a $\Gamma$-homomorphism $\Psi_{d}: F \rightarrow B \rtimes F, x \mapsto(d x, x)$ and, according to the equivalence in Theorem 2.2, the epimorphism $q$ defines a $\Gamma$-group epimorphism 
$Q: A \rtimes F \rightarrow B \rtimes F,(a, x) \mapsto(q(a), x)$. Since $F$ is a free $\Gamma$-group, hence projective, there is a $\Gamma$-homomorphism $\Phi: F \rightarrow A \rtimes F$ such that $Q \Phi=\Psi_{d}$. Then, the $\Gamma$ derivation associated to $\Phi, d_{\Phi}: F \rightarrow A$, by the isomorphism in Proposition (2.5), verifies that $q d_{\Phi}=d$. Therefore, $q_{*}: \operatorname{Der}_{\Gamma}(F, A) \rightarrow \operatorname{Der}_{\Gamma}(F, B)$ is surjective.

It remains to prove that, for each $n \geqslant 1, C_{\Gamma}^{n}(-, A)$ is a flask sheaf on ${ }_{\Gamma} \mathcal{G} / G$, which, by [23, Chapter II, 5, Proposition 1], is equivalent to proving that, for any $\Gamma$-epimorphism $P \stackrel{p}{\rightarrow} Q$ in ${ }_{\Gamma} \mathcal{G} / G$, the Czech complex

$$
0 \rightarrow C_{\Gamma}^{n}(Q, A) \stackrel{p^{*}}{\rightarrow} C_{\Gamma}^{n}(P, A) \stackrel{d^{1}}{\rightarrow} C_{\Gamma}^{n}\left(P \times_{Q} P, A\right) \stackrel{d^{2}}{\rightarrow} C_{\Gamma}^{n}\left(P \times_{Q} P \times_{Q} P, A\right) \rightarrow \cdots
$$

whose coboundary maps are $d^{k}=\sum_{i=1}^{k+1}(-1)^{i+1}\left(p r_{1}, \cdots, p r_{i-1}, p r_{i+1}, \cdots, p r_{k+1}\right)^{*}$, is exact. For, observe that for any $\Gamma$-group over $G, H \rightarrow G$, the abelian group $C_{\Gamma}^{n}(H, A)$ depends only on the pointed underlying set of $H$. Actually, we can define $C_{\Gamma}^{n}(X, A)$ for any pointed set $\left(X, x_{0}\right)$ by

$$
\begin{gathered}
C_{\Gamma}^{n}(X, A)=\bigcup_{p+q=n-1}\left\{f: X^{p+1} \times \Gamma^{q} \rightarrow A \mid f\left(x_{1}, \cdots, x_{p+1}, \sigma_{1}, \cdots \sigma_{q}\right)=0\right. \\
\text { if some } \left.x_{i}=x_{0} \quad \text { or } \quad \sigma_{i}=1\right\},
\end{gathered}
$$

so that the sheaf $C_{\Gamma}^{n}(-, A):{ }_{\Gamma} \mathcal{G} / G \rightarrow \mathcal{A} b$ factors through the obvious forgetful functor $_{\Gamma} \mathcal{G} / G \rightarrow \mathcal{S e t}_{*},(H \rightarrow G) \mapsto H$. Then, given the $\Gamma$-epimorphism $P \stackrel{p}{\rightarrow} Q$, we can choose a pointed map $s: Q \rightarrow P$ right inverse for $p$, and find a contracting homotopy for the Czech complex (16) defined by the homomorphisms $\left(s p p r_{1}, p r_{1}, \cdots, p r_{k}\right)^{*}$, $k \geqslant 0$. Therefore, (16) is exact.

Equivalence of (9) and (10): it follows from [23, Chapter II, 5, Theorem 5]. Alternatively, a direct proof consists in proving that (8) is a flask resolution of the sheaf $\operatorname{Der}_{\Gamma}(-, A)$. Indeed, the exactness of (8) follows from the fact that the cotriple cohomology of a free $\Gamma$-group vanishes in the dimension $\geqslant 1$ ([1, Proposition 2.1$]$ and, for each $n \geqslant 1, \operatorname{Der}_{\Gamma}\left(\mathbb{G}^{n}(-), A\right)$ is flask, since for any $\Gamma$-epimorphism in ${ }_{\Gamma} \mathcal{G} / G$, $P \stackrel{p}{\rightarrow} Q$, the associated Czech complex

$$
0 \rightarrow \operatorname{Der}_{\Gamma}\left(\mathbb{G}^{n}(Q), A\right) \stackrel{p^{*}}{\rightarrow} \operatorname{Der}_{\Gamma}\left(\mathbb{G}^{n}(P), A\right) \stackrel{d^{1}}{\rightarrow} \operatorname{Der}_{\Gamma}\left(\mathbb{G}^{n}\left(P \times_{Q} P\right), A\right) \stackrel{d^{2}}{\rightarrow} \cdots,
$$

whose coboundary maps are $d^{k}=\sum_{i=1}^{k+1}(-1)^{i+1} \mathbb{G}^{n}\left(p r_{1}, \cdots, p r_{i-1}, p r_{i+1}, \cdots, p r_{k+1}\right)^{*}$, has a contracting homotopy given by the homomorphisms $\mathbb{G}^{n-1} \mathbb{F}\left(s p p r_{1}, p r_{1}, \cdots, p r_{k}\right)^{*}$, where $s: Q \rightarrow P$ is any set-section of $p$.

\section{Some properties of the cohomology groups $H_{\Gamma}^{n}(G, A)$.}

The more basic properties of the cohomology are immediate consequences, taking into account Theorem 3, of its definition. We summarize them in the following five points:

a) $H_{\Gamma}^{0}(G, A)=0$.

b) $H_{\Gamma}^{1}(G, A)=\operatorname{Der}_{\Gamma}(G, A)$. 
c) $H_{\Gamma}^{n}(G, I)=0$ for all $n \geqslant 2$, whenever $I$ is an injective $\Gamma$-equivariant $G$-module.

d) $H_{\Gamma}^{n}(F, A)=0$ for all $n \geqslant 2$, whenever $F$ is a free $\Gamma$-group.

e) Any short exact sequence $0 \rightarrow A \rightarrow B \rightarrow C \rightarrow 0$ of $\Gamma$-equivariant $G$-modules provides a long exact sequence

$$
\cdots H_{\Gamma}^{n}(G, A) \rightarrow H_{\Gamma}^{n}(G, B) \rightarrow H_{\Gamma}^{n}(G, C) \rightarrow H_{\Gamma}^{n+1}(G, A) \rightarrow \cdots
$$

There is also an interesting 5 -term exact sequence in the cohomology groups induced by a short exact sequence in the first variable. The paradigm here is the 5 -term exact sequence by Hochschild and Serre [16] for group cohomology.

Suppose that $1 \rightarrow N \stackrel{i}{\rightarrow} E \stackrel{p}{\rightarrow} G \rightarrow 1$ is a short exact sequence of $\Gamma$-groups, thus $N$ can be identified with a normal $\Gamma$-subgroup of $E$ and $E / N \cong G$ as $\Gamma$-groups. Then, the abelianized group $N^{a b}=N /[N, N]$ becomes both a $\Gamma$-module and a $G$-module with actions

$$
\begin{aligned}
& \sigma_{\bar{u}}=\bar{\sigma} \bar{u}, \quad \sigma \in \Gamma, u \in N, \\
& { }^{x} \bar{u}=\overline{e u e^{-1}}, \quad x \in G, u \in N, e \in p^{-1}(x) .
\end{aligned}
$$

Furthermore, since ${ }^{\sigma}\left(e u e^{-1}\right)={ }^{\sigma} e^{\sigma} u^{\sigma} e^{-1}$ and $p\left({ }^{\sigma} e\right)={ }^{\sigma} p(e)$, it follows that ${ }^{\sigma}\left({ }^{x} \bar{u}\right)={ }^{\left({ }^{\sigma} x\right)}\left({ }^{\sigma} \bar{u}\right), \sigma \in \Gamma, x \in G, u \in N$. Hence $N^{a b}$ is a $\Gamma$-equivariant $G$-module.

Theorem 3.1. Let $1 \rightarrow N \stackrel{i}{\rightarrow} E \stackrel{p}{\rightarrow} G \rightarrow 1$ be a short exact sequence of $\Gamma$-groups. Then, for any $\Gamma$-equivariant $G$-module $A$, there is a natural exact sequence

$$
0 \rightarrow H_{\Gamma}^{1}(G, A) \stackrel{p^{*}}{\rightarrow} H_{\Gamma}^{1}(E, A) \stackrel{\gamma}{\rightarrow} \operatorname{Hom}_{\Gamma, G}\left(N^{a b}, A\right) \stackrel{\omega}{\rightarrow} H_{\Gamma}^{2}(G, A) \stackrel{p^{*}}{\rightarrow} H_{\Gamma}^{2}(E, A)
$$

in which $\gamma$ is induced by restricting $\Gamma$-derivations $E \rightarrow A$ to $N$.

Proof. First we prove that there is a natural exact sequence

$$
0 \rightarrow \operatorname{Der}_{\Gamma}(G, A) \stackrel{p^{*}}{\rightarrow} \operatorname{Der}_{\Gamma}(E, A) \stackrel{\gamma}{\rightarrow} \operatorname{Hom}_{\Gamma, G}\left(N^{a b}, A\right)
$$

in which the last map is induced by restriction from $E$ to $N$. Indeed, if $d: E \rightarrow A$ is any $\Gamma$-derivation, then, for all $u \in N$ and $e \in E, d(u e)=d u+d e$ and $d(e u)=$ $d e+{ }^{p(e)} d u$. Hence, the restriction of $d$ to $N$, say $d_{N}: N \rightarrow A$, is a $\Gamma$-homomorphism satisfying $d_{N}\left(e u e^{-1}\right)={ }^{p(e)} d_{N}(u)$, and therefore the induced $\gamma(d)=\overline{d_{N}}: N^{a b} \rightarrow A$, $\bar{u} \mapsto d u$, is both of $\Gamma$-modules and of $G$-modules. If $\gamma(d)=0$, then $x \mapsto d(e)$, $x \in G, e \in p^{-1}(x)$, well defines a $\Gamma$-derivation from $G$ to $A$, which is mapped by $p^{*}$ to $d$; whence the exactness of (18) follows.

Second, we observe that if $p: E \rightarrow G$ admits a $\Gamma$-group section, then the homomorphism $\gamma$ in (18) is also a retraction, that is, (18) is a split short exact sequence of abelian groups. In effect, in such a case, $E=N \rtimes G$ is a semidirect product group with $\Gamma$-action ${ }^{\sigma}(u, x)=\left({ }^{\sigma} u,{ }^{\sigma} x\right)$. Then, the composed map $\xi: N \rtimes G \rightarrow N \rightarrow N^{a b},(u, x) \mapsto \bar{u}$, is a $\Gamma$-derivation that defines a homomorphism $\xi_{*}: \operatorname{Hom}_{\Gamma, G}\left(N^{a b}, A\right) \rightarrow \operatorname{Der}_{\Gamma}(E, A), f \mapsto \xi f$, satisfying $\gamma \xi_{*}=i d$.

Then, we are ready to complete the proof as follows. For each $k \geqslant 0$, let $N_{k}=$ $\operatorname{Ker}\left(\mathbb{G}^{k+1}(p)\right)$ so that $1 \rightarrow N_{\bullet} \rightarrow \mathbb{G}_{\bullet}(E) \rightarrow \mathbb{G}_{\bullet}(G) \rightarrow 1$ is a short exact sequence of simplicial $\Gamma$-groups. Since every $\mathbb{G}^{k}(p), k \geqslant 1$, is a retraction, we obtain a weakly split short exact sequence of cochain complexes

$$
0 \rightarrow \operatorname{Der}_{\Gamma}\left(\mathbb{G}_{\bullet}(G), A\right) \stackrel{p^{*}}{\rightarrow} \operatorname{Der}_{\Gamma}\left(\mathbb{G}_{\bullet}(E), A\right) \stackrel{p^{*}}{\rightarrow} \operatorname{Hom}_{\Gamma, G_{\bullet}(G)}\left(N_{\bullet}^{a b}, A\right) \rightarrow 0,
$$


and the 5-term exact sequence (17) follows from the induced exact sequence in cohomology, proving previously that $H^{0}\left(\operatorname{Hom}_{\Gamma, \mathbb{G} \bullet(G)}\left(N_{\bullet}^{a b}, A\right)\right)=\operatorname{Hom}_{\Gamma, G}\left(N^{a b}, A\right)$.

To do so, let us note that the augmentations $\delta_{E}: \mathbb{G}_{\bullet}(E) \rightarrow E$ and $\delta_{G}: \mathbb{G}_{\bullet}(G) \rightarrow G$ are both homotopy equivalences of Kan simplicial sets [1, Proposition 5.3], and therefore the $N \bullet \rightarrow N$ induced on fibers is also a homotopy equivalence (see [23, Chaper II, 3, Proposition 1]. In particular, $N_{1} \rightrightarrows N_{0} \rightarrow N$ is a right-exact sequence of $\Gamma$-groups, and therefore $N_{1}^{a b} \rightrightarrows N_{0}^{a b} \rightarrow N^{a b} \rightarrow 0$ is also a right-exact sequence of abelian groups. Hence,

$$
\begin{aligned}
H^{0}\left(\operatorname{Hom}_{\Gamma, \mathbb{G}_{\bullet}(G)}\left(N_{\bullet}^{a b}, A\right)\right) & =\operatorname{Ker}\left(\operatorname{Hom}_{\Gamma, \mathbb{G}(G)}\left(N_{0}^{a b}, A\right) \rightarrow \operatorname{Hom}_{\Gamma, \mathbb{G}^{2}(G)}\left(N_{1}^{a b}, A\right)\right) \\
& =\operatorname{Hom}_{\Gamma, G}\left(\operatorname{coker}\left(N_{1}^{a b} \rightarrow N_{0}^{a b}\right), A\right) \\
& =\operatorname{Hom}_{\Gamma, G}\left(N^{a b}, A\right),
\end{aligned}
$$

where we have taken into account that $\mathbb{G}^{2}(G) \rightrightarrows \mathbb{G}(G) \rightarrow G$ is a right-exact sequence of $\Gamma$-groups.

We should note that an explicit description, in terms of cocycles, of the homomorphism $\omega$ in the sequence (17) is as follows: Let $s: G \rightarrow E$ be a set-section of $p$ with $s(1)=1$. Then, for each $\varphi \in \operatorname{Hom}_{\Gamma, G}\left(N^{a b}, A\right), \omega(\varphi) \in H_{\Gamma}^{2}(G, A)$ is the cohomology class of the 2-cocycle $f_{\varphi}: G^{2} \cup(G \times \Gamma) \rightarrow A$ defined by

$$
\begin{array}{ll}
f_{\varphi}(x, y)=\varphi\left(\overline{s(x) s(y) s(x)^{-1}}\right), & x, y \in G, \\
f_{\varphi}(x, \sigma)=\varphi\left(\overline{\sigma s(x) s\left({ }^{\sigma} x\right)^{-1}}\right), & x \in G, \quad \sigma \in \Gamma .
\end{array}
$$

Of course, one can prove that $f_{\varphi}$ is actually a 2-cocycle whose cohomology class in $H_{\Gamma}^{2}(G, A)$ is independent of the choice of $s$, so that $\omega$ is a well-defined homomorphism, and then to prove directly that (17) is exact, but the proof of all of this in full is tedious.

In the hypothesis of Theorem 3.1, the elements of the group of homomorphisms

$$
\left\{\varphi: N \rightarrow A \mid \varphi\left(e u e^{-1}\right)={ }^{p(e)} \varphi(u)\right\} \cong \operatorname{Hom}_{G}\left(N^{a b}, A\right)
$$

were called operator homomorphisms of $N$ in $A$ by MacLane [18]; then, it is natural to call the elements of its subgroup,

$$
\left\{\varphi: N \rightarrow A \mid \varphi\left(e u e^{-1}\right)={ }^{p(e)} \varphi(u), \varphi\left({ }^{\sigma} u\right)={ }^{\sigma} \varphi(u)\right\} \cong \operatorname{Hom}_{\Gamma, G}\left(N^{a b}, A\right),
$$

$\Gamma$-equivariant operator homomorphisms (or operator $\Gamma$-homomorphisms) of $N$ in $A$. Each $\Gamma$-derivation of $E$ in $A$, if restricted to $N$, yields an operator $\Gamma$-homomorphism $N \rightarrow A$. By the group $\operatorname{Map}_{\Gamma}(N, E ; A)$ of operator $\Gamma$-homomorphism classes of $N$ into $A$, we understand the group of all operator $\Gamma$-homomorphisms $\varphi: N \rightarrow A$, module the subgroup of those operator $\Gamma$-homomorphisms induced by $\Gamma$-derivations of $E$ into $A$. Therefore,

$$
\operatorname{Map}_{\Gamma}(N, E ; A) \cong \operatorname{Hom}_{\Gamma, G}\left(N^{a b}, A\right) / \gamma\left(H_{\Gamma}^{1}(G, A)\right) .
$$

The following is an equivariant version of a classic result by MacLane $[\mathbf{1 8}$, Theorems A, A'], which was the counterpart to Hopf's formula for group cohomology.

Theorem 3.2. If $R$ is a normal $\Gamma$-subgroup of the free $\Gamma$-group $F, G \cong F / R$ and $A$ is a $\Gamma$-equivariant $G$-module, then the group $\operatorname{Map}_{\Gamma}(R, F ; A)$ depends only on the 
$\Gamma$-groups $G$ and $A$ and the operators of $G$ on $A$ and not on the chosen representation of $G$ by the free $\Gamma$-group $G$. Furthermore, there is a natural isomorphism

$$
\operatorname{Map}_{\Gamma}(R, F ; A) \cong H_{\Gamma}^{2}(G, A) .
$$

Proof. Consider the 5 -term exact sequence (17) induced by $R \longmapsto F \rightarrow G$. Since $F$ is a free $\Gamma$-group $H_{\Gamma}^{2}(F, A)=0$, and therefore $\operatorname{Map}_{\Gamma}(R, F ; A) \cong \operatorname{coker}(\gamma) \stackrel{\omega}{\cong}$ $H_{\Gamma}^{2}(G, A)$.

Suppose now that

$$
\underline{E}: A \stackrel{i}{\longmapsto} E \stackrel{p}{\rightarrow} G,
$$

is a short exact sequence of $\Gamma$-groups in which $A$ is abelian, that is, $A$ is a $\Gamma$ module. Then $A^{a b}=A$ is a $\Gamma$-equivariant $G$-module with the $G$-action defined by the equality $i\left({ }^{x} a\right)=e i(a) e^{-1}, x \in G, a \in A, e \in p^{-1}(x)$. We define a $\Gamma$ group extension of the $\Gamma$-group $G$ by the $\Gamma$-equivariant $G$-module $A$ as a short exact sequence of $\Gamma$-groups (21) such that the $G$-module structure induced on $A$ is the given $G$-module structure. We say the extension $\underline{E}$ is equivalent to $\underline{E^{\prime}}$ if there exists a $\Gamma$-group isomorphism $\Phi: E \cong E^{\prime}$ such that $\Phi i=i^{\prime}$ and $p^{\prime} \Phi=p$. We denote by

$$
E_{\Gamma}(G, A)
$$

the set of equivalence classes of $\Gamma$-group extensions of $G$ by $A$. Next we prove that there is a one-to-one correspondence between $E_{\Gamma}(G, A)$ and the cohomology group $H_{\Gamma}^{2}(G, A)$. This result does not come as a surprise. The category of $\Gamma$-groups is tripleable over $\mathcal{S} e t$; hence, Beck's theorem $[\mathbf{3}]$ shows that $H_{\Gamma}^{2}(G, A)$ classifies the principal $\Gamma$-groups over $G$ under $A$ (or under $A \rtimes G \rightarrow G$ ), that is, $A$-torsors over $G$. Then, we could proceed, similarly as for groups (see [3, Example 4]), verifying that principal $\Gamma$-groups over $G$ under $A$ are equivalent to $\Gamma$-group extensions of $G$ by $A$. This is a roundabout way of proving the classification theorem that we shall establish directly bellow (see [6] for another proof using a factor set theory for $\Gamma$-group extensions).

Theorem 3.3. Let $G$ be $a \Gamma$-group and let $A$ be a $\Gamma$-equivariant $G$-module. Then there is a natural bijection

$$
H_{\Gamma}^{2}(G, A) \cong E_{\Gamma}(G, A) .
$$

Proof. Given isomorphism (20), the proof is parallel to the proof in [15, Theorem 10.3] for group cohomology, except for the fact that the constructions needed are $\Gamma$-equivariant. Thus, given a free $\Gamma$-group presentation of $G, R \longmapsto F \stackrel{q}{\rightarrow} G$, and an extension (21), choose a map $\theta: F \rightarrow G$ of $\Gamma$-groups over $G$. Then, the restriction of $\theta$ to kernels $\left.\theta\right|_{R}: R \rightarrow A$ is an operator $\Gamma$-homomorphism of $R$ into $A$, whose class in $\operatorname{Map}_{\Gamma}(R, F ; A)$ is independent of the choice of $\theta$. This defines a map $\Phi: E_{\Gamma}(G, A) \rightarrow$ $\operatorname{Map}_{\Gamma}(R, F ; A)$. Conversely, given a $\Gamma$-equivariant operator homomorphism $\varphi: R \rightarrow$ $A$ let $E_{\varphi}=(A \rtimes F) / U$, where $A \rtimes F$ is the $\Gamma$-group defined by the semidirect product group of $A$ and $F$ with $\Gamma$-action ${ }^{\sigma}(a, x)=\left({ }^{\sigma} a,{ }^{\sigma} x\right)$, and $U=\left\{\left(\varphi(r), r^{-1}\right), r \in R\right\}$. Observe that $U$ is a normal $\Gamma$-subgroup of $E_{\varphi}$. The sequence $A \stackrel{i}{\longmapsto} E_{\varphi} \stackrel{\pi}{\rightarrow} G$, 
$i(a)=\overline{(a, 1)}, \pi(\overline{(a, x)})=q(x)$, is easily seen to be a $\Gamma$-group extension of $G$ by $A$, and it is straightforward to verify that this procedure gives an inverse to $\Phi$.

We should note that the cohomology groups $H_{\Gamma}^{3}(G, A)$ appear in the classification of $\Gamma$-group extensions with a non-abelian kernel (see [26] and [6, Theorem 5.1]). Further, an interpretation of these cohomology groups $H_{\Gamma}^{3}(G, A)$ is given in $[\mathbf{6}]$, where degree three equivariant cohomology classes are expressed in terms of graded monoidal categories. Note that there is already a general interpretation of cotriple cohomology by Duskin [7], which applies to our cohomology groups $H_{\Gamma}^{n}(G, A), n \geqslant$ 1, by Theorem 2.6.

Next we prove that $H_{\Gamma}^{n}(-, A)$, considered as a functor of the first variable, are coproduct-preserving, that is, the cohomology of the free product of two $\Gamma$-groups is the direct sum of the cohomologies of each of them.

If $G_{1}$ and $G_{2}$ are $\Gamma$-groups, let $G_{1} * G_{2}$ denote their coproduct. If $H$ is any $\Gamma$-group, $G_{1} * G_{2}$ is characterized by a natural isomorphism

$$
\operatorname{Hom}_{\Gamma} \mathcal{G}\left(G_{1} * G_{2}, H\right) \cong \operatorname{Hom}_{\Gamma} \mathcal{G}\left(G_{1}, H\right) \times \operatorname{Hom}_{\Gamma} \mathcal{G}\left(G_{2}, H\right) .
$$

Then, for any $\Gamma$-module $A$,

$$
\operatorname{Hom}_{\Gamma} \mathcal{G}\left(G_{1} * G_{2}, \operatorname{Aut}(A)\right) \cong \operatorname{Hom}_{\Gamma} \mathcal{G}\left(G_{1}, \operatorname{Aut}(A)\right) \times \operatorname{Hom}_{\Gamma} \mathcal{G}\left(G_{2}, \operatorname{Aut}(A)\right),
$$

and, by Theorem 2.2 , it follows that $A$ is a $\Gamma$-equivariant $\left(G_{1} * G_{2}\right)$-module if and only if $A$ is simultaneously a $\Gamma$-equivariant $G_{1}$ - and $G_{2}$-module.

Theorem 3.4. Let $G_{1}, G_{2}$ be two $\Gamma$-groups and let $A$ be a $\Gamma$-equivariant $\left(G_{1} * G_{2}\right)$ module, then the coproduct injections yield isomorphisms

$$
H_{\Gamma}^{n}\left(G_{1} * G_{2}, A\right) \cong H_{\Gamma}^{n}\left(G_{1}, A\right) \oplus H_{\Gamma}^{n}\left(G_{2}, A\right) .
$$

Proof. It is quite similar to that by Barr and Rinehart [2, Theorem 4.1] for the cohomology of a free product of groups. If $G$ is any $\Gamma$-group and $U \subset G$ is any $\Gamma$-subgroup of $G$, then every injective $\Gamma$-equivariant $G$-module is, by restriction, an injective $\Gamma$-equivariant $U$-module $(U \rtimes \Gamma$ is a subgroup of $G \rtimes \Gamma$ and therefore an injective $(G \rtimes \Gamma)$-module is an injective $(U \rtimes \Gamma)$-module [15, Corollary 1.4], then use Theorem 2.6). Applying this to the situation under consideration, we see that there is a simultaneous $\Gamma$-equivariant $G_{1^{-}}, G_{2^{-}}$and $\left(G_{1} * G_{2}\right)$-injective resolution, say $I^{\bullet}$, of $A$. From (24) and the isomorphism in Proposition 2.5, we obtain

$$
\operatorname{Der}_{\Gamma}\left(G_{1} * G_{2}, I^{\bullet}\right) \cong \operatorname{Der}_{\Gamma}\left(G_{1}, I^{\bullet}\right) \oplus \operatorname{Der}_{\Gamma}\left(G_{2}, I^{\bullet}\right),
$$

and passing to cohomology this yields the desired result.

The following properties deal with the relationship between the cohomology of $\Gamma$-groups and the ordinary cohomology groups.

If $\Gamma=\mathbf{1}$, the trivial group, then a $\mathbf{1}$-group is the same as a group, and for each group $G$, a 1 -equivariant $G$-module is simply an ordinary $G$-module. 
Theorem 3.5. Let $G$ be a group and let $A$ be a $G$-module, then

$$
H_{1}^{n}(G, A)= \begin{cases}\operatorname{Der}(G, A) & \text { if } n=1 \\ H^{n}(G, A) & \text { if } n \geqslant 2,\end{cases}
$$

where $H^{\bullet}(G, A)$ is the ordinary group cohomology.

Proof. It is immediate from the definition and theorem (2.6).

Let us recall from Theorem 2.2 that, for any $\Gamma$-group $G$, a $\Gamma$-equivariant $G$-module is the same as a $(G \rtimes \Gamma)$-module. In the following theorem we show a fundamental relationship between the cohomology groups $H_{\Gamma}^{n}(G, A)$ with the usual cohomology groups $H^{n}(G \rtimes \Gamma, A)$ and $H^{n}(\Gamma, A)$, by means of a long exact sequence linking these groups. We should stress that the projection $G \rtimes \Gamma \rightarrow \Gamma$ induces, in general, no homomorphism $H^{n}(\Gamma, A) \rightarrow H^{n}(G \rtimes \Gamma, A)$ (see Corollary 3.7 below).

Theorem 3.6. Let $G$ be a $\Gamma$-group and let $A$ be a $\Gamma$-equivariant $G$-module. Then there is a natural long exact sequence

$$
\begin{aligned}
& 0 \rightarrow H^{0}(G \rtimes \Gamma, A) \rightarrow H^{0}(\Gamma, A) \rightarrow H_{\Gamma}^{1}(G, A) \rightarrow H^{1}(G \rtimes \Gamma, A) \rightarrow \cdots \\
& \cdots \rightarrow H_{\Gamma}^{n}(G, A) \rightarrow H^{n}(G \rtimes \Gamma, A) \rightarrow H^{n}(\Gamma, A) \rightarrow H_{\Gamma}^{n+1}(G, A) \rightarrow \cdots
\end{aligned}
$$

Proof. After Theorem 2.6, the above long exact sequence is an immediate consequence of definition (12) for $H_{\Gamma}^{n}(G, A)$, because the exactness property of cohomology with local coefficients. For an algebraic proof, we can proceed as follows. Firstly, observe the existence of the 4-term truncated exact sequence, that is, of the exact sequence

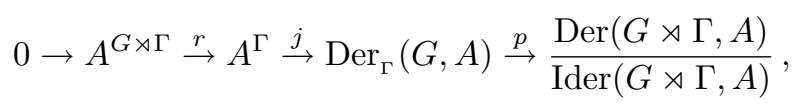

where $r$ is the inclusion map, $j$ is the homomorphism mapping each $\Gamma$-equivariant element $a \in A^{\Gamma}$ to the inner $\Gamma$-derivation from $G$ into $A, j_{a}: x \mapsto{ }^{x} a-a$, and $p$ is the homomorphism defined by $p(d)=\bar{D}$, where $D(x, \sigma)=x$.

Since $H^{1}(G \rtimes \Gamma, I)=0$ if $I$ is any injective $\Gamma$-equivariant $G$-module, from (26) it follows that the sequence of left-exact functors from the category of $\Gamma$-equivariant $G$-modules to the category of abelian groups

$$
0 \rightarrow H^{0}(G \rtimes \Gamma,-) \rightarrow H^{0}(\Gamma,-) \rightarrow H_{\Gamma}^{1}(G,-) \rightarrow 0
$$

is exact on injectives. Then, (25) is the corresponding long exact sequence of derived functors.

A $\Gamma$-equivariant $G$-module on which $G$ acts trivially is simply a $\Gamma$-module that is considered a $(G \rtimes \Gamma)$-module via the projection $G \rtimes \Gamma \rightarrow \Gamma$. For these coefficients we have the following consequence of Theorem 3.6.

Corollary 3.7. Let $G$ be $a \Gamma$-group and let $A$ be a $\Gamma$-equivariant trivial $G$-module, that is, a $\Gamma$-module. Then there are natural isomorphisms,

$$
H^{n}(G \rtimes \Gamma, A) \cong H_{\Gamma}^{n}(G, A) \oplus H^{n}(\Gamma, A) .
$$


Proof. The hypothesis of $A$ being a trivial $G$-module implies that the restriction maps $H^{n}(G \rtimes \Gamma, A) \stackrel{r}{\rightarrow} H^{n}(\Gamma, A)$ are retractions, with sections induced by the projection $G \rtimes \Gamma \rightarrow \Gamma$. Then, the long exact sequence (25) gives split exact sequences

$$
0 \longrightarrow H_{\Gamma}^{n}(G, A) \longrightarrow H^{n}(G \rtimes \Gamma, A) \underset{\gtrless}{\rightleftarrows} H^{n}(\Gamma, A) \longrightarrow 0 .
$$

The following consequence is for ordinary group cohomology.

Corollary 3.8. If $F$ is any free $\Gamma$-group, then

$$
H^{n}(F \rtimes \Gamma, A) \cong H^{n}(\Gamma, A)
$$

for all $(F \rtimes \Gamma)$-module $A$ and all $n \geqslant 2$.

Proof. In the corresponding long exact sequence $(25), H_{\Gamma}^{n}(F, A)=0$ for all $n \geqslant 2$, whence the restriction maps $H^{n}(F \rtimes \Gamma, A) \rightarrow H^{n}(\Gamma, A)$ are isomorphisms.

If $G$ is a $\Gamma$-group and $A$ is any $\Gamma$-equivariant $G$-module, the usual group of derivations from $G$ into $A, \operatorname{Der}(G, A)$, is canonically a $\Gamma$-module by the diagonal $\Gamma$-action $\left({ }^{\sigma} d\right)(x)={ }^{\sigma} d\left({ }^{\sigma^{-1}} x\right), \sigma \in \Gamma, d \in \operatorname{Der}(G, A), x \in G$, so that $\operatorname{Der}(G,-)$ is a functor from the category of $\Gamma$-equivariant $G$-modules into the category of $\Gamma$ modules,

$$
\operatorname{Der}(G,-):{ }_{\Gamma, G} \mathcal{A} b \rightarrow{ }_{\Gamma} \mathcal{A} b .
$$

Lemma 3.9. Let $G$ be a $\Gamma$-group. If $I$ is an injective $\Gamma$-equivariant $G$-module, then $\operatorname{Der}(G, I)$ is an injective $\Gamma$-module.

Proof. By Corollary $2.3 \mathrm{ii}), I$ is an injective $G$-module, then $H^{1}(G, I)=0$ and therefore every derivation of $G$ in $I$ is an inner derivation. Let $j: I \rightarrow \operatorname{Der}(G, I)$ be the homomorphism that maps every $a \in I$ to the inner derivation $j_{a}: x \mapsto{ }^{x} a-a$. Since $I$ is a $\Gamma$-equivariant $G$-module, $j$ is a $\Gamma$-module homomorphism. In fact,

$$
\left.\left({ }^{\sigma} j_{a}\right)(x)={ }^{\sigma} j_{a}\left({ }^{\sigma^{-1}} x\right)={ }^{\sigma}\left({ }^{\left(\sigma^{-1}\right.} x\right) a-a\right)={ }^{x}\left({ }^{\sigma} a\right)-{ }^{\sigma} a=j_{\sigma} a .
$$

Hence $0 \rightarrow I^{G} \rightarrow I \stackrel{j}{\rightarrow} \operatorname{Der}(G, A) \rightarrow 0$ is a short exact sequence of $\Gamma$-modules. Since, again by Corollary 2.3, both $I$ and $I^{G}$ are injective $\Gamma$-modules, it follows that the above exact sequence splits and that $\operatorname{Der}(G, A)$ is an injective $\Gamma$-module.

We are now ready to prove the following relationship between the cohomology of groups $\Gamma$ and $G$ and those of $G$ as a $\Gamma$-group.

Theorem 3.10. Let $G$ be a $\Gamma$-group and let $A$ be a $\Gamma$-equivariant $G$-module. Then, there is a natural action of $\Gamma$ on the cohomology groups $H^{n}(G, A)$, given by the homomorphism $\Gamma \rightarrow \operatorname{Aut}\left(H^{n}(G, A)\right), \sigma \mapsto \sigma_{*}^{-1} \sigma^{*}$, and a spectral sequence $\left\{E_{n}\right\}$ such that

$$
E_{2}^{p, q}=\left\{\begin{array}{ll}
H^{p}\left(\Gamma, H^{q+1}(G, A)\right) & \text { if } q \geqslant 1 \\
H^{p}(\Gamma, \operatorname{Der}(G, A)) & \text { if } q=0,
\end{array} \Longrightarrow H_{\Gamma}^{p+q+1}(G, A),\right.
$$

which converges finitely to the graded group associated with $\left\{H_{\Gamma}^{n}(G, A)\right\}$, suitably filtered. 
Proof. The spectral sequence (28) is deduced from the Grothendieck composite functor spectral sequence [14], when it is applied to the following commutative diagram of functors:

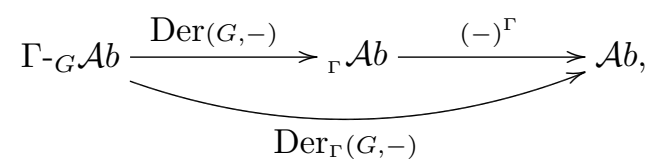

taking into account that $\operatorname{Der}(G,-)$ preserves injectives, by Lemma 3.9. Indeed, the Grothendieck spectral sequence is

$$
E_{2}^{p, q}=\left(R^{p}(-)^{\Gamma}\right)\left(R^{q} \operatorname{Der}(G,-)\right)(A) \Longrightarrow\left(R^{p+q} \operatorname{Der}_{\Gamma}(G,-)\right)(A) .
$$

Since, by Corollary 2.3, an injective $\Gamma$-equivariant $G$-module resolution of $A$ is also an injective $G$-module resolution of $A$, then

$$
R^{q} \operatorname{Der}(G,-)(A)= \begin{cases}H^{q+1}(G, A) & q \geqslant 1 \\ \operatorname{Der}(G, A) & q=0\end{cases}
$$

whence

$$
E_{2}^{p, q}= \begin{cases}H^{p}\left(\Gamma, H^{q+1}(G, A)\right) & q \geqslant 1 \\ H^{p}(\Gamma, \operatorname{Der}(G, A)) & q=0 .\end{cases}
$$

Furthermore, $R^{p+q} \operatorname{Der}_{\Gamma}(G,-)(A)=H_{\Gamma}^{p+q+1}(G, A)$ by definition (3), so that spectral sequence (29) is identified with (28).

Alternatively, we can obtain the spectral sequence (28) from the double cochain complex $C_{\Gamma}^{\bullet \bullet}(G, A)$ defined before definition $(7)$ of $H_{\Gamma}^{n}(G, A)$.

Since Theorem 3.6, we have been amassing evidence that the cohomology groups $H_{\Gamma}^{n}(G, A)$ are closely related to the ordinary cohomology groups of the semidirect product group $H^{n}(G \rtimes \Gamma, A)$. The spectral sequence (28) is another indication of that fact, because the Lyndon-Hochschild-Serre spectral sequence [16]

$$
E_{2}^{p, q}=H^{p}\left(\Gamma, H^{q}(G, A)\right) \Rightarrow H^{p+q}(G \rtimes \Gamma, A) .
$$

Any group can be regarded as a trivial $\Gamma$-group. If $G$ is a group and $A$ is a $G$ module, then $A$ is a $\Gamma$-equivariant $G$-module with the trivial action of $\Gamma$ on both $G$ and $A$; so a natural question is to ask whether there is any relationship between the cohomology groups $H^{n}(G, A)$ and $H_{\Gamma}^{n}(G, A)$. The varietal Baer invariants theory (cf., e.g. [10],[12],[22],[4]) allows us to provide some answers to this problem. Indeed, the category of groups, $\mathcal{G}$, is a Birkoff subvariety of the category of $\Gamma$-groups, ${ }_{\Gamma} \mathcal{G}$, and the corresponding quotient (reflector) functor is

$$
Q:{ }_{\Gamma} \mathcal{G} \rightarrow \mathcal{G} p, \quad G \mapsto G /[\Gamma ; G],
$$

where for any $\Gamma$-group $G,[\Gamma ; G]$ is the normal $\Gamma$-subgroup of $G$ generated by the elements of the form ${ }^{\sigma} x x^{-1}, x \in G, \sigma \in \Gamma$. The Baer invariants $B_{n}(G), n \geqslant 0$, of a $\Gamma$-group $G$ are defined as $B_{n}(G)=L_{n} Q(G)$, where $L_{n} Q$ is the (non-abelian) $n$-th derived functor of $R$, so that they can be computed from the cotriple resolution of $G, \mathbb{G}_{\bullet} \rightarrow G$, as the (Moore) homotopy groups of the simplicial group $Q \mathbb{G} \bullet(G)$; that 
is,

$$
B_{n}(G)=\pi_{n}\left(\frac{\mathbb{G}_{\bullet}(G)}{\left[\Gamma ; \mathbb{G}_{\bullet}(G)\right]}\right), \quad n \geqslant 0 .
$$

Hence, $B_{0}(G)=Q(G)=\frac{G}{[\Gamma ; G]}$ and each $B_{n}(G), n \geqslant 1$, is a $B_{0}(G)$-module. The invariant $B_{1}(G)$ is the most commonly studied one in the literature; there is a "Hopf" formula to compute it from any free presentation of the $\Gamma$-group $G[\mathbf{1 2}]$, $R \longmapsto F \rightarrow G$, namely

$$
B_{1}(G)=\frac{R \cap[\Gamma ; F]}{\left[\Gamma ; R_{1} F\right]},
$$

where $\left[\Gamma ; R_{1} F\right]$ is the normal $\Gamma$-subgroup of $F$ generated by the elements of the form $u^{\sigma} x^{\sigma} v v^{-1} x^{-1} u^{-1} x^{\sigma} x^{-1}, u, v \in R, x \in F, \sigma \in \Gamma$, on which the action of $\frac{G}{[\Gamma ; G]}$ is induced by conjugation in $F$.

Observe that if $G$ is a $\Gamma$-group, then a $\Gamma$-equivariant $G$-module $A$ on which $\Gamma$ acts trivially is the same as a $\frac{G}{[\Gamma ; G]}$-module. Then, by specialization of Theorem 9 in [4], we have the following theorem:

Theorem 3.11. Let $G$ be a $\Gamma$-group and let $A$ be $a \frac{G}{[\Gamma ; G]}$-module. Then,

$$
H_{\Gamma}^{1}(G, A) \cong \operatorname{Der}\left(\frac{G}{[\Gamma ; G]}, A\right),
$$

and there exists an exact sequence of abelian groups

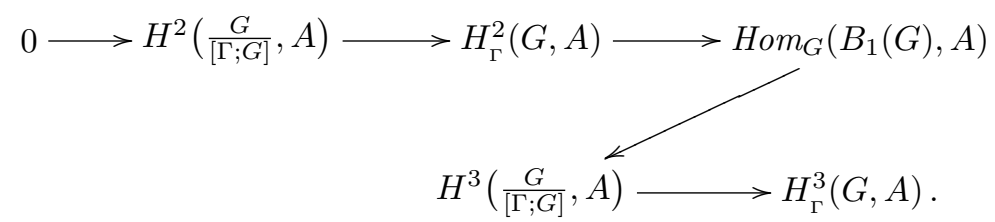

Moreover, if $B_{i}(G)=0$ for all $1 \leqslant i<n$, then

$$
H_{\Gamma}^{i}(G, A) \cong H^{i}\left(\frac{G}{[\Gamma ; G]}, A\right), \quad 2 \leqslant i \leqslant n,
$$

and there exists an exact sequence of abelian groups

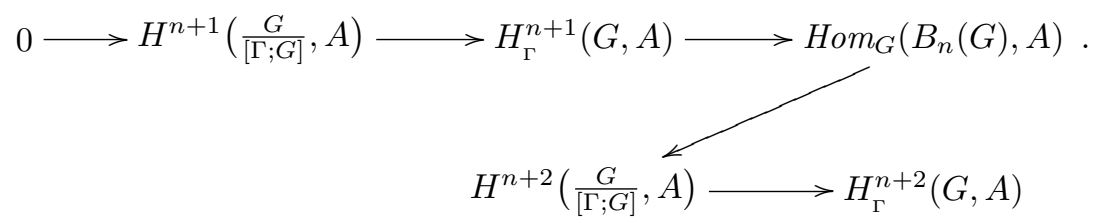

The category of $\Gamma$-modules is also a Birkoff subvariety of the category of $\Gamma$-groups. In this case, the quotient functor is the abelianization functor

$$
()^{a b}:{ }_{\Gamma} \mathcal{G} p \rightarrow{ }_{\Gamma} \mathcal{A} b, \quad G \mapsto G^{a b}=G /[G, G],
$$

and the corresponding Baer invariants of a $\Gamma$-group $G$ are the homotopy groups of the simplicial $\Gamma$-module $\mathbb{G}_{\bullet}(G)^{a b}$. Since every free $\Gamma$-group is also a free group, it is easy to see that $\mathbb{G}_{\bullet}(G)$ is a free simplicial group with $\pi_{0}\left(\bar{G}_{\bullet}(G)\right)=G$ and 
$\pi_{i}\left(\bar{G}_{\bullet}(G)\right)=0$ for all $i>0$ (recall that the augmented simplicial set $\mathbb{G}_{\bullet}(G) \rightarrow G$ has a canonical contraction and therefore $\mathbb{G}_{\bullet}(G)$ is a loop group complex of the Eilenberg-MacLane complex $K(G, 1))$. Hence, by $([\mathbf{2 1}]$, Theorem 26.9$), \pi_{n} \mathbb{G}_{\bullet}(G)^{a b}=$ $H_{n+1}(G), n \geqslant 0$, the integral homology of group $G$ at dimension $n+1$, with the induced $\Gamma$-module structure by the $\Gamma$-action on $G$.

Then, again by specialization of Theorem 9 in $[4]$, we obtain the following theorem:

Theorem 3.12. Let $G$ be $a \Gamma$-group and let $A$ be $a \Gamma$-module which we consider as a $\Gamma$-equivariant $G$-module on which $G$ acts trivially. Then,

$$
H_{\Gamma}^{1}(G, A)=H_{\Gamma}\left(G^{a b}, A\right),
$$

and there exists an exact sequence of abelian groups

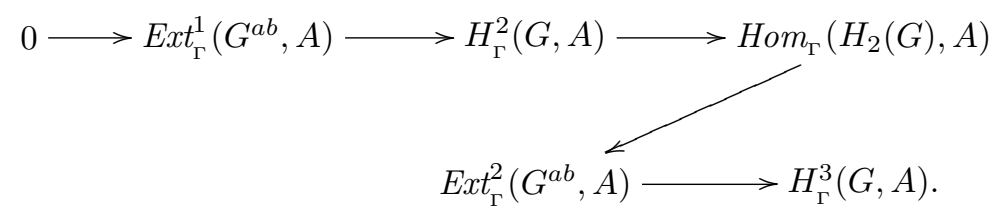

Moreover, if $H_{i}(G)=0$ for all $1 \leqslant i<n$, then

$$
H_{\Gamma}^{i}(G, A) \cong \operatorname{Ext}_{\Gamma}^{i-1}\left(G^{a b}, A\right), \quad 2 \leqslant i \leqslant n,
$$

and there exists an exact sequence of abelian groups

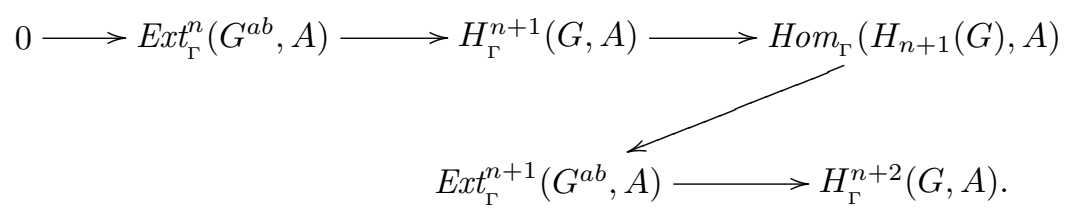

This theorem can also be obtained from the "universal coefficients" spectral sequence

$$
\operatorname{Ext}_{\Gamma}^{p}\left(H_{q+1}(G), A\right) \Rightarrow H_{\Gamma}^{p+q+1}(G, A)
$$

which, for any $\Gamma$-group $G$ and any $\Gamma$-module $A$, considered as a trivial $G$-module, can be deduced [24, Theorem 2.26] from the following commutative diagram of functors:

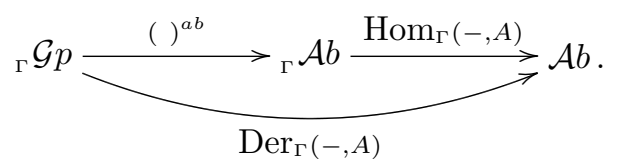

If, in Theorem 3.12, the $\Gamma$-group $G$ is such that $H_{i}(G)=0$ for all $i \geqslant 2$, then it follows that there are isomorphisms

$$
H_{\Gamma}^{n+1}(G, A) \cong \operatorname{Ext}_{\Gamma}^{n}\left(G^{a b}, A\right),
$$

for all $n \geqslant 0$ and every $\Gamma$-module $A$, regarded as a trivial $G$-module. For instance, we can take $G=\mathbb{Z}$, the group of integers with the trivial $\Gamma$-action. In this case, 
$\operatorname{Ext}_{\Gamma}^{n}\left(\mathbb{Z}^{a b}, A\right)=\operatorname{Ext}_{\Gamma}^{n}(\mathbb{Z}, A)=H^{n}(\Gamma, A)$ for every $\Gamma$-module $A$, so we deduce the following consequence.

Corollary 3.13. Let $\mathbb{Z}$ be the group of integers considered as a trivial $\Gamma$-group and let $A$ be any $\Gamma$-module, considered as a $\Gamma$-equivariant $\mathbb{Z}$-module on which group $\mathbb{Z}$ acts trivially. Then, there are natural isomorphisms

$$
H_{\Gamma}^{n+1}(\mathbb{Z}, A) \cong H^{n}(\Gamma, A), \quad n \geqslant 0 .
$$

Isomorphism (31) can be combined with the one in Corollary 3.7, which yields the following curious conclusion on ordinary group cohomology.

Corollary 3.14. Let $A$ be $a \Gamma$-module and consider $A$ as a $(\mathbb{Z} \times \Gamma)$-module via the projection $\mathbb{Z} \times \Gamma \rightarrow \Gamma$. Then, there are natural isomorphisms

$$
H^{n}(\mathbb{Z} \times \Gamma, A) \cong H^{n-1}(\Gamma, A) \oplus H^{n}(\Gamma, A)
$$

for all $n \geqslant 0\left(H^{-1}(\Gamma, A)=0\right)$.

Actually, Corollary 3.14 is known when $A$ is a trivial $\Gamma$-module: in that case, the Künneth theorem gives $H_{n}(\mathbb{Z} \times \Gamma)=H_{n}(\Gamma) \oplus H_{n-1}(\Gamma)$ and isomorphism (31) follows from universal coefficients.

Corollary 3.13 can be generalized to the case in which the $\Gamma$-action on $\mathbb{Z}$ is not trivial and $A$ is an arbitrary $\mathbb{Z}$-module as in the proposition below.

Proposition 3.15. Suppose that $\mathbb{Z}$ is endowed with a $\Gamma$-group structure by a homomorphism $\rho: \Gamma \rightarrow A u t(\mathbb{Z})=\{i d,-i d\}$. Then, for any $\Gamma$-equivariant $\mathbb{Z}$-module $A$, there are isomorphisms

$$
H_{\Gamma}^{n+1}(\mathbb{Z}, A) \cong H^{n}\left(\Gamma, A^{\star}\right), \quad n \geqslant 0,
$$

where $A^{\star}$ denotes the $\Gamma$-module that is the same abelian group $A$ with action from $\Gamma$ by

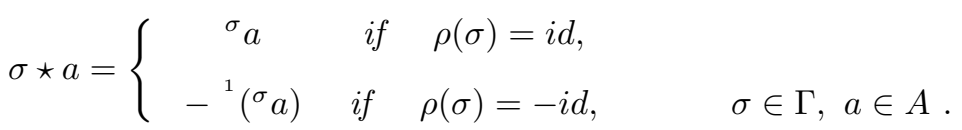

Proof. Since $H^{q+1}(\mathbb{Z}, A)=0$ for all $q \geqslant 1$, the spectral sequence (28) degenerates, giving isomorphisms $H^{n}(\Gamma, \operatorname{Der}(\mathbb{Z}, A)) \cong H_{\Gamma}^{n+1}(\mathbb{Z}, A)$, where $\operatorname{Der}(\mathbb{Z}, A)$ is a $\Gamma$ module with the diagonal action $\left({ }^{\sigma} d\right)(m)={ }^{\sigma} d\left({ }^{\sigma^{-1}} m\right)$. Now, the map $d \mapsto d(1)$ is easily recognized as being a $\Gamma$-module isomorphism $\operatorname{Der}(\mathbb{Z}, A)=A^{\star}$, whence the isomorphisms (33) follow.

The above Proposition 3.15 can be used to show some explicit computations of equivariant cohomology groups. For example, let $C_{2 k}=<t \mid t^{2 k}=1>$ denote the cyclic group of order $2 k$ generated by $t$, and consider the $C_{2 k}$-action on the group of integers $\mathbb{Z}$ such that ${ }^{t} m=-m$ and the action of $\mathbb{Z}$ on itself given by ${ }^{n} m=(-1)^{n} \cdot m$. Then, $\mathbb{Z}$ is an equivariant module over the $C_{2 k}$-group $\mathbb{Z}$ and, by Proposition 3.15 , $H_{C_{2 k}}^{n+1}(\mathbb{Z}, \mathbb{Z}) \cong H^{n}\left(C_{2 k}, \mathbb{Z}\right)$, whence, by $([\mathbf{1 5}$, VI, Proposition 7.1$])$, we conclude that

$$
H_{C_{2 k}}^{n+1}(\mathbb{Z}, \mathbb{Z})= \begin{cases}\mathbb{Z}_{2} & \text { if } n \text { is odd } \\ 0 & \text { if } n \text { is even }\end{cases}
$$


We shall conclude by exhibiting another example of computation, in this case using the complexes $C_{\Gamma}^{\bullet}(G, A)$. For let us consider the cyclic group of order two $C_{2}$ acting trivially on itself, and the action of $C_{2}$ on $\mathbb{Z}$ by ${ }^{t} m=-m$. Then $\mathbb{Z}$ is an equivariant module over the $C_{2}$-group $C_{2}$ and the cohomology groups $H_{C_{2}}^{n}\left(C_{2}, \mathbb{Z}\right)$ are defined.

Observe that, in this case, a normalized $n$-cochain

$$
f: \bigcup_{p+q=n-1} C_{2}^{p+1} \times C_{2}^{q} \longrightarrow \mathbb{Z}
$$

is determined by the sequence of $n$ integers $\left(a_{1}, \ldots, a_{n}\right) \in \mathbb{Z}^{n}$, where

$$
a_{i}=f(\overbrace{t, \ldots, t}^{n-i+1} ; \overbrace{t, \ldots, t}^{i-1}), \quad 0 \leqslant i \leqslant n,
$$

so that $C_{C_{2}}^{n}\left(C_{2}, \mathbb{Z}\right) \cong \mathbb{Z}^{n}$ and the coboundary operator of $C_{C_{2}}^{\bullet}\left(C_{2}, \mathbb{Z}\right)$ can be rewritten as the maps $\partial^{n}: \mathbb{Z}^{n} \rightarrow \mathbb{Z}^{n+1}$ given by $\partial\left(a_{1}, \ldots, a_{n}\right)=\left(b_{1}, \ldots, b_{n+1}\right)$, where

$$
\begin{aligned}
& b_{1}=\left(-1+(-1)^{n+1}\right) a_{1} \\
& b_{k}=\left(-1+(-1)^{k-1}\right) a_{k-1}+\left((-1)^{k}+(-1)^{n+1}\right) a_{k}, \quad 2 \leqslant k \leqslant n \\
& b_{n+1}=\left(-1+(-1)^{n}\right) a_{n} .
\end{aligned}
$$

In the case in which $n$ is odd, say $n=2 m-1$, then a $(2 m-1)$-cochain $\left(a_{1}, \ldots, a_{2 m-1}\right)$ is a cocycle if and only if $a_{k-1}=a_{k}, 2 \leqslant k \leqslant 2 m-2$, and $a_{2 m-1}=0$. Furthermore, such a cocycle is a coboundary if and only if every $a_{i}$ is even. Then, we conclude that

$$
H_{C_{2}}^{2 m-1}\left(C_{2}, \mathbb{Z}\right) \cong \mathbb{Z}_{2}^{m-1} .
$$

On the other hand, when $n$ is even, say $n=2 m$, a $2 m$-cochain $\left(a_{1}, \ldots, a_{2 m}\right)$ is a cocycle if and only if $a_{i}=0$ whenever $i$ is odd and, furthermore, such a cocycle is a coboundary if and only if every $a_{i}$ is even. Hence,

$$
H_{C_{2}}^{2 m}\left(C_{2}, \mathbb{Z}\right) \cong \mathbb{Z}_{2}^{m}
$$

\section{References}

[1] M. Barr and J. Beck, Homology and standard constructions, in B. Eckmann, editor, Seminar on Triples and Categorical Homology Theory, Lecture Notes in Math. 80, Springer-Verlag, (1969).

[2] M. Barr and G. Rinehart, Cohomology as the derived functor of derivations. Trans. Amer. Math. Soc. 122(1966), 416-426.

[3] J. Beck, Triples, Algebras and Cohomology, (1967), Dissertation submitted to Columbia University.

[4] A.M. Cegarra and M. Bullejos, Cohomology and Higher dimensional Baer invariants, J. of Algebra 132 (1990), 321-339. 
[5] A.M. Cegarra and L. Fernández, Cohomology of cofibred categorical groups, J. of Pure and Appl. Algebra 143 (1999), 107-154.

[6] A.M. Cegarra, J.M. García-Calcines and J.A. Ortega, On graded categorical groups and equivariant group extensions, Canad. J. Math., to appear.

[7] J. Duskin, Simplicial methods and the interpretation of triple cohomology, Mem. AMS 3, 163, 1975.

[8] A. Dold and D. Puppe, Homologie nicht-additiver Funktoren, Anwendungen, Ann. Inst. Fourier 11 (1961), 201-312.

[9] S. Eilenberg and S. Mac Lane, Cohomology theory in abstract groups, I, Ann. of Math. 48 (1947), 51-78.

[10] A. Fröhlich, Non-Abelian homological algebra II. Varieties, Proc. London Math. Soc. (3) 11 (1962), 1-28.

[11] A. Fröhlich and C.T.C. Wall, Graded monoidal categories, Compos. Math. 28 (1974), 229-285.

[12] J. Furtado-Coelho, Homology and generalized Baer invariants, J. of Algebra 40 (1976), 596-609.

[13] P.G. Goerss and P.G. Jardine, Simplicial homotopy theory, P. M. 174, Birkhäuser, 1999.

[14] A. Grothendieck, Sur quelques points d'algèbre homologique, Tôhoku Math. J. 9 (1957), 119-221.

[15] P.J. Hilton and U. Stammbach, A course in homological algebra, Grad. Texts in Math. 4, Springer, 1971.

[16] G. Hochschild and P. Serre, Cohomology of group extensions, Trans. Amer. Math. Soc. 74 (1953), 110-134.

[17] P.T. Johnstone, Topos theory, Academic Press, 1977.

[18] S. MacLane, Cohomology theory in abstract groups,III. Ann. of Math. 50 (1949). 736-761.

[19] S. MacLane, Homology, Die Grundleheren der Math. Wiss. in Einzel. 114, Springer, 1963.

[20] S. MacLane, Categories for the working mathematician, Grad. Texts in Math. 5, Springer, 1971.

[21] J.P. May, Simplicial objects in algebraic topology, Van Nostrand, 1967.

[22] K. Mody, Simplicial methods and the homology of groups, (1976). Dissertation submitted to London University.

[23] D. Quillen, Homotopical algebra, Lecture Notes in Math. 43, Springer-Verlag, (1967).

[24] G. Rinehart, Satellites and cohomology, J. of Algebra 12 (1969), 295-329. 
[25] J.H.C. Whitehead, Combinatorial homotopy II, Bull. Amer. Math. Soc 55 (1949), 469-543.

[26] J.H.C. Whitehead, On Group extensions with operators, Quart. J. Math. Oxford 2 (1950), 219-228.

This article may be accessed via WWW at http://www.rmi.acnet.ge/hha/ or by anonymous ftp at

ftp://ftp.rmi.acnet.ge/pub/hha/volumes/2002/n1a1/v4n1a1.(dvi,ps,pdf)

A. M. Cegarra acegarra@ugr.es

Departamento de Álgebra

Universidad de Granada

Spain

Granada

Facultad de Ciencias, 18071 Granada

J. M. García-Calcines jmgarcal@ull.es

Departamento de Matemática Fundamental

Universidad de La Laguna

Spain

City

38271, La Laguna, Tenerife

J. A. Ortega jalcaraz@ugr.es

Departamento de Álgebra

Universidad de Granada

Spain

Granada

Facultad de Ciencias, 18071 Granada 